Reconstruction (ASiR-V), which eliminated background noise with BMI calculated adjustments.

Methods All scans were prospectively gated with zero padding. BMI was calculated and radiation dose adjusted accordingly. We collected retrospective data for coronary CT Angiograms that excluded repeat scans due to technical errors as well as graft studies to ensure uniformity. Data gathered included patient demographics, body mass index and exam dose-length product (DLP).

Results A total of 110 coronary CTA exams performed 1 year after the survey were randomly selected for analysis in our study. The median BMI and exam DLP were $27.7 \mathrm{~kg} / \mathrm{m}^{2}$ and $138 \mathrm{mGycm}$, respectively. The median exam DLP in our previous study was $194 \mathrm{mGycm}$. Therefore, the median exam DLP using the newer machines and reconstruction technique is lower by $28.86 \%$ in comparison to the previous survey. In addition, we observed a trend of higher exam DLP with increasing BMI.

Conclusion Using the newer scanners in combination with ASiR-V at our hospital led to reduced radiation doses.

\section{COMPARISON OF THE ACCURACY OF FLASH VERSUS PROSPECTIVELY ACQUIRED CT CORONARY ANGIOGRAPHY}

Kate Liang, Mark Dayer, Oliver E Gosling. Department of Cardiology, Taunton and Somerset Hospital

\subsection{6/heartjnl-2019-BSCI.8}

Introduction Computed Tomography Coronary Angiography (CTCA) is an accurate and established diagnostic tool. The development of ECG triggered spiral acquisition (FLASH) has allowed reduction in radiation dose whilst still providing diagnostic information. We aimed to compare the accuracy of FLASH and prospective CTCA acquisitions within our CT service.

Method Retrospective analysis of demographic and outcome data was performed for 2857 scans over a 69 month period (Feb 2013-Nov 2018). Results of CTCA and Invasive Coronary Angiography (ICA) were compared for patients having had FLASH or prospective scan protocols.

Results 289 patients were identified as having had both CTCA and ICA. 55 (19\%) of these studies were FLASH, 173 (59.9\%) prospective, $2(0.7 \%)$ retrospective, 41 (14.2\%) calcium score only, and $18(6.2 \%)$ requiring $\geq 2$ contrast studies. [Results are reported FLASH vs Prospective] Diagnostic scans in $92.7 \%$ vs $93.6 \%$. Mean BMI $\left(\mathrm{Kgm}^{2}\right): 28(22-46)$ vs $30(18-46)$. Mean acquisition HR (bpm): 55 (48-70) vs 59 (41-166, median 59). Median dose metoprolol (mg): $5 \quad(0-30)$ vs 5 (0-50). Median DLP $(\mathrm{mGy} * \mathrm{~cm}): 79(49-351)$ vs $199(60-964)$. There was $58.2 \%$ agreement with FLASH studies and ICA versus $67.1 \%$ agreement with prospective acquisitions. This was not statistically significant $(p=0.26)$.

Conclusion Our data demonstrates that there is no statistically significant difference between FLASH and prospectively gated CTCA studies within our service. There is however a trend demonstrating that FLASH scans are not as accurate as prospectively gated scans although this is in a relatively small patient population. More studies with larger patient numbers and multi-centre analysis are recommended.

\section{DUAL ANTIPLATELET THERAPY TO INHIBIT MYOCARDIAL INJURY IN PATIENTS WITH HIGH-RISK CORONARY ARTERY PLAQUE: A RANDOMISED CONTROLLED TRIAL}

${ }^{1}$ Alastair J Moss, 'Marc R Dweck, 'Mhairi K Doris, '1Jack PM Andrews, 'Rong Bing, ${ }^{1}$ Rachael 0 Forsythe, ${ }^{1}$ Timothy R Cartlidge, ${ }^{1}$ Tania A Pawade, ${ }^{1}$ Marwa Daghem, ${ }^{2}$ Jennifer B Raftis, ${ }^{1,3}$ Michelle C Williams, ${ }^{1,3}$ Edwin JR van Beek, ${ }^{4}$ Laura Forsyth, ${ }^{4}$ Steff $C$ Lewis, ${ }^{4}$ Robert Lee, ${ }^{1,5}$ Anoop SV Shah, ${ }^{1,5}$ Nicholas L Mills, ${ }^{1,3}$ David E Newby, ${ }^{1,6}$ Philip D Adamson. ${ }^{1}$ British Heart Foundation Centre for Cardiovascular Science, University of Edinburgh, Edinburgh, UK; ${ }^{2}$ Medical Research Council Centre for Inflammation Research, University of Edinburgh, Edinburgh, UK; ${ }^{3}$ Edinburgh Imaging, Queen's Medical Research Institute University of Edinburgh, Edinburgh, UK; ${ }^{4}$ Edinburgh Clinical Trials Unit, University of Edinburgh, Edinburgh, UK; ${ }^{5}$ Usher Institute of Population Health Sciences and Informatics, University of Edinburgh, UK; ${ }^{6}$ Christchurch Heart Institute, University of Otago, Christchurch, New Zealand

\subsection{6/heartjnl-2019-BSCI.9}

Introduction High-risk coronary atherosclerotic plaque is associated with higher plasma troponin concentrations suggesting ongoing myocardial injury that may be a target for dual antiplatelet therapy. The aim of this study is to determine whether ticagrelor reduces high-sensitivity troponin I concentrations in patients with established coronary artery disease and high-risk coronary plaque using coronary 18F-fluoride positron emission tomography-computed tomography.

Methods In a randomised double-blind placebo-controlled trial, patients with multivessel coronary artery disease underwent coronary $18 \mathrm{~F}$-fluoride positron emission tomography-computed tomography and measurement of high-sensitivity cardiac troponin I and were randomised (1:1) to ticagrelor $90 \mathrm{mg}$ twice daily or matched placebo. The primary endpoint was troponin I concentration at 30 days in patients with increased coronary 18F-fluoride uptake.

Results In total, 202 patients were randomized and 191 met the pre-specified criteria for inclusion in the primary analysis. In patients with increased coronary 18F-fluoride uptake $(n=120 / 191)$ there was no evidence that ticagrelor had an effect on plasma troponin concentrations at 30 days (ratio of geometric means for ticagrelor versus placebo, 1.11, [95\% confidence interval 0.90 to 1.36 ], $\mathrm{p}=0.32$ ). Over 1 year, ticagrelor had no effect on troponin concentrations in patients with increased coronary 18F-fluoride uptake (ratio of geometric means, $0.86,95 \%$ confidence interval 0.63 to 1.17 , $\mathrm{p}=0.33)$.

Conclusion Dual antiplatelet therapy with ticagrelor does not reduce plasma troponin concentrations in patients with coronary $18 \mathrm{~F}$-fluoride uptake. This suggests that subclinical plaque thrombosis does not contribute to ongoing myocardial injury in this setting.

\section{CAC-RDS AND CAD-RDS AS A POTENTIAL TOOL TO BETTER CHARACTERISE CAD PREVALENCE, SEVERITY AND VARIATION WITHIN UK NICE CG95 COMPLIANT PATHWAYS}

Matthias Schmitt, Abid Ullah, Anna Reid, Craig Caswell, Chris Wong, Gavin Freeman, Melanie Greaves, Gaetano Nucifora. Manchester University NHS Foundation Trust, UK

\subsection{6/heartinl-2019-BSCI.10}

Background CAC-RADS and CAD-RADS were introduced to create a standardized method to communicate findings of coronary calcium scoring (CCS) and coronary CT angiography (cCTA) and to facilitate standardized clinical decision making 